\title{
First-Order 0- $\pi$ Quantum Phase Transition in the Kondo Regime of a Superconducting Carbon-Nanotube Quantum Dot
}

\author{
Romain Maurand, ${ }^{1}$ Tobias Meng, ${ }^{2}$ Edgar Bonet, ${ }^{1}$ Serge Florens, ${ }^{1}$ Laëtitia Marty, ${ }^{1, *}$ and Wolfgang Wernsdorfer ${ }^{1}$ \\ ${ }^{1}$ Institut Néel, CNRS et Université Joseph Fourier, BP 166, F-38042 Grenoble Cedex 9, France \\ ${ }^{2}$ Institut für Theoretische Physik, Universität zu Köln, Zülpicher Strasse 77, 50937 Köln, Germany \\ (Received 2 October 2011; published 15 February 2012; publisher error corrected 16 February 2012)
}

\begin{abstract}
We study a carbon-nanotube quantum dot embedded in a superconducting-quantum-interference-device loop in order to investigate the competition of strong electron correlations with a proximity effect. Depending on whether local pairing or local magnetism prevails, a superconducting quantum dot will exhibit a positive or a negative supercurrent, referred to as a 0 or $\pi$ Josephson junction, respectively. In the regime of a strong Coulomb blockade, the 0 -to- $\pi$ transition is typically controlled by a change in the discrete charge state of the dot, from even to odd. In contrast, at a larger tunneling amplitude, the Kondo effect develops for an odd-charge (magnetic) dot in the normal state, and quenches magnetism. In this situation, we find that a first-order 0 -to- $\pi$ quantum phase transition can be triggered at a fixed valence when superconductivity is brought in, due to the competition of the superconducting gap and the Kondo temperature. The superconducting-quantum-interference-device geometry together with the tunability of our device allows the exploration of the associated phase diagram predicted by recent theories. We also report on the observation of anharmonic behavior of the current-phase relation in the transition regime, which we associate with the two accessible superconducting states. Our results finally demonstrate that the spin-singlet nature of the Kondo state helps to enhance the stability of the 0 phase far from the mixed-valence regime in odd-charge superconducting quantum dots.
\end{abstract}

DOI: 10.1103/PhysRevX.2.011009

\section{INTRODUCTION}

Realizing a Josephson junction with a carbon nanotube as a weak link opened up the way to a new class of nanoelectronic devices that combine both quantum confinement at the nanoscale and the Josephson effect [1-16]. In such a junction, a simplified view would predict a maximum critical current when a discrete electronic level of the quantum dot comes into resonance with the Cooper condensate of the electrodes, thus allowing an electrostatic tuning of the critical current magnitude determined by the quantum-level position [17]. The Josephson effect in a quantum dot is more complex, however, because it is governed by the interplay of electronic pairing and strong Coulomb interaction on the dot [18-28]. When superconductivity dominates, the superconductor wave function spreads over the dot, inducing a BCS-singlet ground state, i.e., a standard Josephson junction (dubbed the 0 state in what follows) [17]. In the other extreme regime of large electron-electron interactions, the quantum dot enters the Coulomb-blockade domain, and its charge is locked to integer values, altering the superconducting state. For an odd occupancy, the quantum dot behaves like a spin $S=1 / 2$ magnetic impurity that competes with

\footnotetext{
*laetitia.marty@grenoble.cnrs.fr

Published by the American Physical Society under the terms of the Creative Commons Attribution 3.0 License. Further distribution of this work must maintain attribution to the author(s) and the published article's title, journal citation, and DOI.
}

Subject Areas: Mesoscopics, Nanophysics, Superconductivity

Cooper-pair formation, and the ground state can become a magnetic doublet. In this situation, dissipationless current mainly transits through a process involving four tunneling events that reorders the spins of Cooper pairs, thus leading to a negative sign of the supercurrent, which is referred to as the $\pi$-type Josephson junction [3,4,29]. The antagonist 0 and $\pi$ superconducting states, associated with a sharp sign reversal of the dissipationless current at zero temperature, can hence allow a first-order quantum phase through tuning of the microscopic parameters in the quantum dot. In the case of a very strong Coulomb blockade, the $0-\pi$ transition is achieved by modifying the parity of the electronic charge on the dot (the valence is easily changed using electrostatic gates), so that the supercurrent sign reversal occurs at the edges of the Coulomb diamonds. A more intriguing regime occurs for intermediate Coulomb repulsion (associated with moderately small values of the tunneling amplitude compared to the charging energy), in which Kondo correlations take place: In the normal state, the magnetic impurity of the odd-charge state is screened through spin-flip cotunneling processes [30], providing a nonzero density of states at the Fermi energy. This so-called Kondo resonance allows the Cooper pairs to flow normally in the superconducting state, and a 0-type Josephson junction is therefore recovered $[15,16,19,23,24]$. Here, we explore in detail how superconducting transport is affected by the presence of Kondo behavior, and we finely tune the $0-\pi$ quantum phase transition in this intermediate Coulomb-repulsion regime by controlling the microscopic parameters of the quantum dot. 


\section{CHARACTERIZATION OF THE NANO-SQUID}

\section{A. Sample fabrication}

In this section, we investigate supercurrent reversal in a carbon-nanotube Josephson junction using the nano-superconducting-quantum-interference-device (nanoSQUID) geometry, which implements two Josephson junctions in parallel built with a single carbon nanotube [4]. The single-wall carbon nanotubes are obtained using laser ablation and then dispersed in a pure dichloroethane solution using low-power ultrasounds. A degenerately phosphorusdoped silicon wafer with a 450-nm layer of $\mathrm{SiO}_{2}$ on top is used as a backgate. The first optical-lithography step provides alignment marks which are then used to locate the nanotubes by scanning electron microscopy. The superconducting loops and the sidegates are fabricated using aligned e-beam lithography, which is followed by e-beam evaporation of the Pd-Al bilayer (with respective thicknesses of $4 \mathrm{~nm}$ and $50 \mathrm{~nm}$ ). All measurements are performed in a dilution refrigerator with a base temperature of $T=$ $35 \mathrm{mK}$, and the filtering stages are similar to the ones performed in Ref. [4]. Samples are current-biased for both DC and lock-in measurements (with an AC amplitude of $10 \mathrm{pA}$ ), so that the switching current or the differential resistance of the device can be measured directly. The nano-SQUID switching currents $I_{\mathrm{sw}}$ are detected via a digital filter that monitors the estimated variance of the average DC voltage. (See Appendix C 1 and Ref. [31].)

Figure 1(a) shows a scanning electron micrograph of the measured nano-SQUID with two 350-nm-long nanotube Josephson junctions (JJ1 and JJ2). Using the second quantum dot as a tunable reference junction, we gain a precise control over the energy $\epsilon_{0}$ and linewidth $\Gamma$ of the first quantum dot by tuning a pair of local sidegates and a backgate $\left(V_{\mathrm{SG} 1}, V_{\mathrm{SG} 2}, V_{\mathrm{BG}}\right.$, respectively); see the discussion in Secs. II B and II C. Such a geometry allows
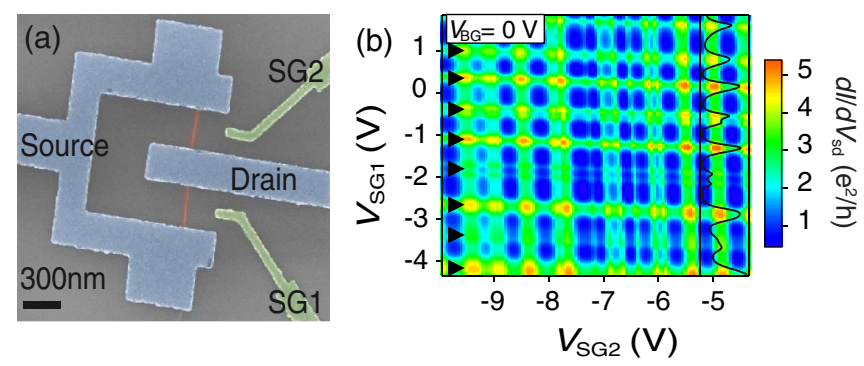

FIG. 1. Nano-SQUID characteristics. (a) Scanning-electronmicroscopy micrograph of the measured nano-SQUID. The two sidegates (SG1, SG2) are green, the nanotube defining two quantum dots is orange, and the superconducting leads are blue. (b) Map of the normal-state zero-bias conductance $d I / d V_{\text {sd }}$ vs the two sidegate voltages at magnetic field $B=75 \mathrm{mT}$, temperature $T=35 \mathrm{mK}$, and backgate voltage $V_{\mathrm{BG}}=0 \mathrm{~V}$. Black triangles indicate the odd-occupancy regions of the first quantum dot (QD1). A line cut of the linear conductance at fixed $V_{\mathrm{SG} 2}=$ $-5.25 \mathrm{~V}$ is also shown, in black. us to directly measure the Josephson current of a single junction via the magnetic-field modulation of the SQUID switching current $I_{\mathrm{sw}}$; see Ref. [4]. Indeed, the critical current of an asymmetric SQUID with a sinusoidal current-phase relation (taken here for simplicity) can be written as

$$
I_{\mathrm{c}}=\sqrt{\left(I_{\mathrm{c} 1}-I_{\mathrm{c} 2}\right)^{2}+4 I_{\mathrm{c} 1} I_{\mathrm{c} 2}\left|\cos \left(\pi \frac{\phi}{\phi_{0}}+\frac{\delta_{1}+\delta_{2}}{2}\right)\right|^{2}},
$$

where $\phi$ is the flux modulation of the SQUID, $\phi_{0}=h / 2 e$ is the magnetic-flux quantum, $\delta_{1}, \delta_{2}$ are the intrinsic phase shifts $(0$ or $\pi)$ of the two Josephson junctions, and $I_{\mathrm{c} 1}, I_{\mathrm{c} 2}$ are the respective critical currents of the junctions. The critical-current modulation is thus shifted by $\phi_{0} / 2$ between the $0-0$ and the $\pi-0$ SQUID configurations.

\section{B. Normal-state transport properties}

Figure 1(b) presents the nano-SQUID stability diagram, where $d I / d V_{\text {sd }}$ at zero bias plotted as a function of $V_{\mathrm{SG} 1}$ and $V_{\mathrm{SG} 2}$ in the normal state (a perpendicular magnetic field of $B=75 \mathrm{mT}$ is applied to suppress superconductivity), at a given backgate $V_{\mathrm{BG}}=0 \mathrm{~V}$. This diagram resembles a slightly tilted checkerboard pattern, which is typical for two parallel, uncoupled quantum dots in the Coulombblockade regime, with a weak crosstalk of about $4 \%$. The linecut at fixed $V_{\mathrm{SG} 2}=-5.25 \mathrm{~V}$ emphasizes the regions of high and low differential conductance associated with the Kondo ridges and Coulomb-blockaded valleys, respectively. One can indeed distinguish easily between even and odd occupancies in each dot from the sequence of conducting and blocked regions: Dark blue pockets denote regimes where both dots are blocked (in an even-even configuration of the double-dot setup); green lines correspond to the situation where one of the dots is in the Kondo regime (see arrows) while the other remains blocked (in an even-odd configuration); and orange spots show the case where both dots undergo the Kondo effect (in an odd-odd configuration) [4,30].

An operating region at a different backgate voltage $V_{\mathrm{BG}}=-0.3 \mathrm{~V}$ is shown in greater detail in Fig. 2(a). For $V_{\mathrm{SG} 1}$ between $1.70 \mathrm{~V}$ and $1.95 \mathrm{~V}$, JJ1 has an odd occupancy associated with a differential conductance close to $2 e^{2} / h$ due to a well-developed Kondo effect. Furthermore, JJ2 clearly has an even number of electrons for $V_{\mathrm{SG} 2}$ between $-4.85 \mathrm{~V}$ and $-5.15 \mathrm{~V}$, because of its small contribution to transport in this range. In order to show the influence of the backgate voltage $V_{\mathrm{BG}}$, we have plotted in Fig. 2(b) the differential conductance vs $V_{\mathrm{SG} 1}$ for the odd-occupancy region of JJ1 corresponding to the white cut in Fig. 2(a), for five different values of $V_{\mathrm{BG}}$ from $-0.3 \mathrm{~V}$ to $-0.7 \mathrm{~V}$. Upon application of $V_{\mathrm{BG}}$, the sidegates 1 and 2 experience a capacitive crosstalk of $-21.5 \%$ and $-17.4 \%$, respectively, as seen by the global 

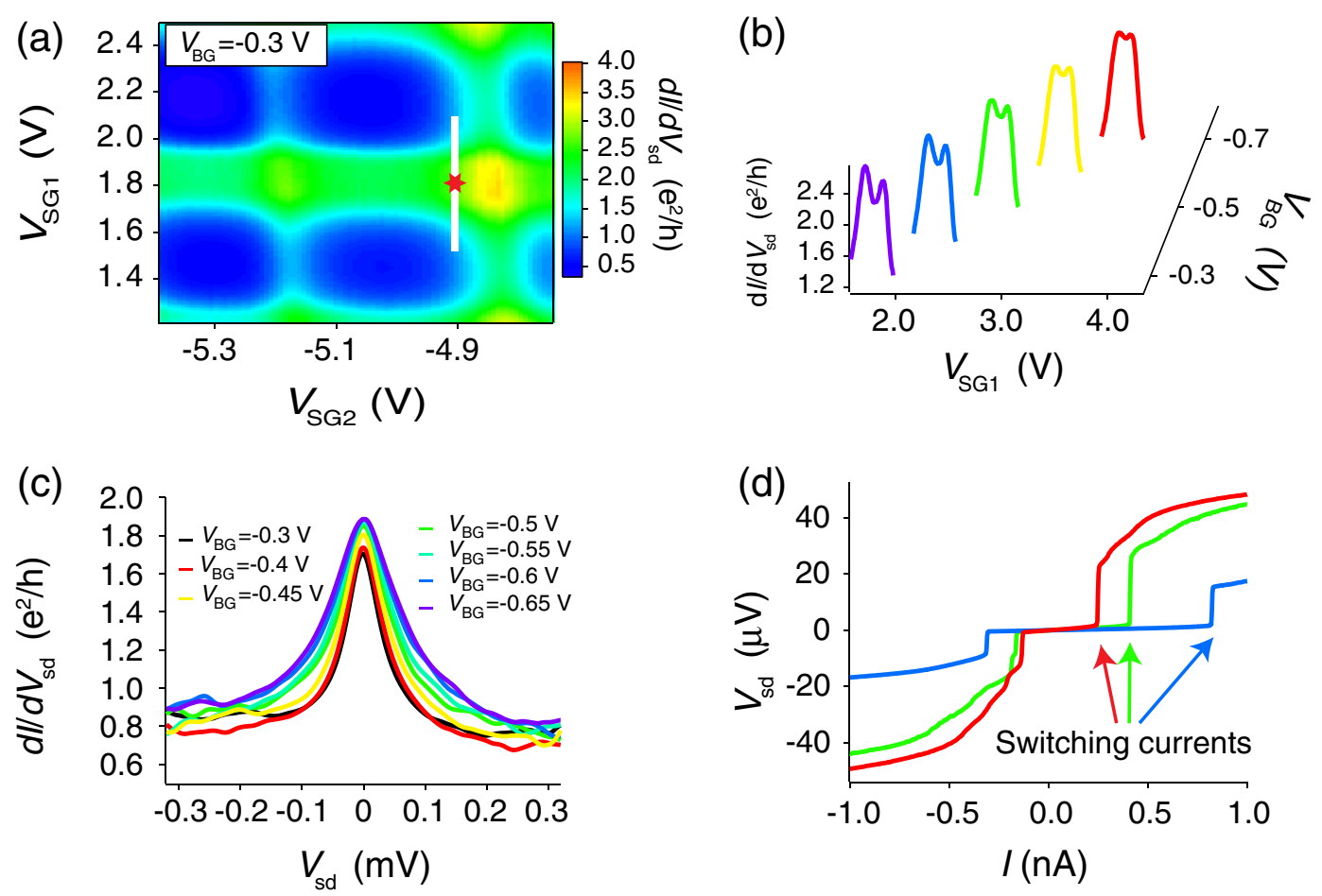

FIG. 2. Kondo correlations and voltage/current characteristics. (a) Zero-bias conductance $d I / d V_{\text {sd }}$ vs the sidegate voltages $V_{\mathrm{SG} 1}$ and $V_{\mathrm{SG} 2}$ in the normal state (under an applied magnetic field $B=75 \mathrm{mT}$ ) at $V_{\mathrm{BG}}=-0.3 \mathrm{~V}$, where JJ1 presents a well-established Kondo ridge for $1.7 \mathrm{~V}<V_{\mathrm{SG} 1}<1.95 \mathrm{~V}$ and JJ2 has an even occupancy for $-5.15 \mathrm{~V}<V_{\mathrm{SG} 2}<-4.85 \mathrm{~V}$. (b) Zero-bias $d I / d V_{\mathrm{sd}}$ conductance vs sidegate voltage $V_{\mathrm{SG} 1}$ in the normal state along the white line in panel (a) for five backgate voltages $V_{\mathrm{BG}}$ between $-0.3 \mathrm{~V}$ and $-0.7 \mathrm{~V} . V_{\mathrm{SG} 2}$ has been corrected for crosstalk in order to follow the white line, but $V_{\mathrm{SG} 1}$ is shown as measured. (c) Finite-bias differential conductance $d I / d V_{\text {sd }}$ vs source-drain voltage $V_{\text {sd }}$ in the normal state, taken in the middle of the Kondo ridge of JJ1 [red star in panel (a), corresponding to a level position $\epsilon_{0}=0$ ] for seven backgate voltages $V_{\mathrm{BG}}$ between $-0.7 \mathrm{~V}$ and $-0.3 \mathrm{~V}$. The width of the Kondo resonance is modified by the backgate voltage while $\epsilon_{0}$ and $U$ are kept constant, implying a variation of $\Gamma$. (d) Typical superconducting voltage-current characteristics of the nano-SQUID for three arbitrary values of the gate voltages $\left(V_{\mathrm{SG} 1}, V_{\mathrm{SG} 2}\right)$. The data are analyzed throughout the paper by recording the switching currents $I_{\mathrm{sw}}$ obtained from such voltage-current plots; see Appendix C 1 for information on the experimental technique.

shifts of the conductance traces. The application of a backgate voltage thus modifies not only the occupation number on the dot, but also the tunnel linewidth $\Gamma$ $[11,32]$. Indeed, by varying the backgate voltage and correcting the voltages of the sidegates for crosstalk, it is possible to keep the local Coulomb repulsion $U$ and the level position $\epsilon_{0}$ on the quantum dots relatively constant [16], while the hybridization $\Gamma$ of the first quantum dot (QD1) to the leads experiences sizeable variations up to about $20 \%$ as we discuss next.

\section{Tuning the hybridization with the gates}

In Fig. 2(c), the Kondo resonances taken in the middle of the odd-occupancy region of QD1 [see corresponding red star in Fig. 2(a)] are superimposed for different values of $V_{\mathrm{BG}}$. The hybridization $\Gamma$ can be extracted for different values of the backgate voltage $V_{\mathrm{BG}}$ from the half-width at half-maximum $V_{\mathrm{K}}$ of the Kondo resonance in the finitebias conductance. In order to systematically extract $V_{\mathrm{K}}$, we use a Lorentzian line shape with a fixed background corresponding to the QD2 contribution to transport and to a small elastic cotunneling component for QD1 [33]. Qualitatively, we note the clear increase of $V_{\mathrm{K}}$ that is achieved by shifting the backgate voltage to more negative values, which is related to the gate-induced enhancement of the hybridization $\Gamma$ reported above. More precisely, in the scaling limit of the Kondo problem [34], a universal behavior of all physical observables is obtained as a function of the Kondo scale, here expressed as a Kondo voltage $V_{\mathrm{K}}$,

$$
V_{\mathrm{K}}=\alpha \sqrt{\Gamma U} \exp \left[\frac{-\pi U}{8 \Gamma}\left(1-4 \frac{\epsilon_{0}^{2}}{U^{2}}\right)\right],
$$

with $U$ the Coulomb repulsion on the dot, $\Gamma$ its total hybridization to the leads, and $\epsilon_{0}$ its energy shift (taken by convention to be zero in the middle of the diamond). This expression applies in the limit of $U \gg \Gamma$ (for $-U / 2<\epsilon_{0}<U / 2$ ), and contains a yet-undetermined prefactor $\alpha$, which depends on the coupling regime $U / \Gamma$. To obtain the value of the charging energy $U$, we have considered the Coulomb-stability diagram of JJ1 (see Appendix B 1) and extrapolated the diamond edges to 
large bias. Because of the large linewidth of our strongly coupled nanostructure, the determination of $U$ leads to moderate error bars, and our estimate is $U=0.80 \pm$ $0.05 \mathrm{meV}$. Focusing on the differential conductance $d I / d V_{\text {sd }}$ from now on, we set a more precise definition of $\alpha$ by our choice of $V_{\mathrm{K}}$ as the half-width at half-maximum of the finite-bias Kondo peak. In a nearequilibrium situation (corresponding to a very asymmetric barrier to the left and right leads) and in the regime where $U \gtrsim 6 \Gamma$, we find that the unknown parameter is given by $\alpha^{\mathrm{eq}} \simeq 2.8$ from numerical renormalization-group calculations [35]. However, in our experiment, the conductance is tuned to its maximum value of $4 e^{2} / h$ (i.e., $2 e^{2} / h$ per dot), corresponding to an equally balanced tunneling amplitude from each of the leads. (We note that values slightly above $4 e^{2} / h$ can be achieved in our double-dot device, which we attribute to small extra elastic contributions from symmetry-broken orbital states of the carbon nanotube.) In that case of symmetrically coupled quantum dots, decoherence of the Kondo anomaly is induced by the antagonist pinning of the Kondo resonance to the split Fermi levels of each lead, reducing the half-width $V_{\mathrm{K}}$ of the finite-bias conductance peak compared to the equilibrium situation. For the relevant regime $U \gtrsim 6 \Gamma$, one can estimate [36] a reduction by $50 \%$ of the linewidth, so that we finally fix $\alpha=1.4$. Because there is to date no fully controlled theory of the finite-bias Kondo resonance, we believe that the imprecise choice of $\alpha$ will introduce the largest error in our determination of $\Gamma$, and hence of the phase boundary to be analyzed in Sec. III B. The final backgate dependence of

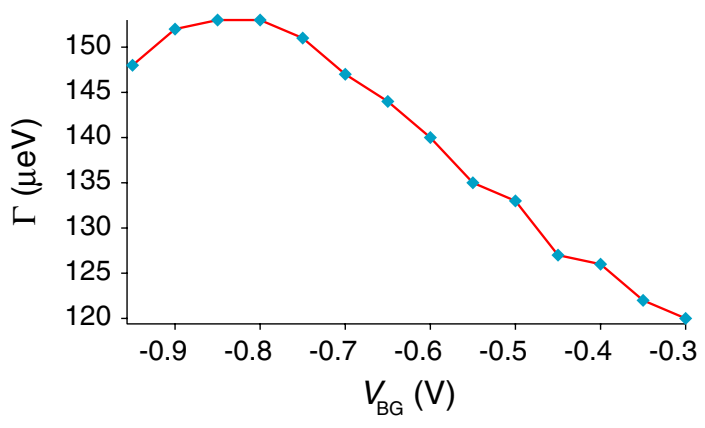

FIG. 3. Total hybridization $\Gamma$ vs backgate voltage $V_{\mathrm{BG}}$. Blue diamonds represent the determined $\Gamma$ from measured $V_{\mathrm{K}}$ at different backgate voltages. This determination is performed in the middle of the Coulomb diamond of JJ1; see text for details. A control with $20 \%$ amplitude variations of $\Gamma$ is thus achieved by tuning the backgate. For visibility, error bars are not indicated here, but they are plotted in Fig. 6(a). Clearly, the backgatevoltage variation of the hybridization is not monotonous and can affect the left-right symmetry of the hybridization of the QD as well. This backgate-voltage range $(-0.3 \mathrm{~V}$ to $-0.7 \mathrm{~V})$ is chosen in order to minimize the asymmetric change of the hybridization, which is a subleading effect compared to the exponential change of the Kondo temperature associated with the variations of $\Gamma$. the hybridization $\Gamma$ is shown in Fig. 3 for the middle point (particle-hole symmetric) of the Coulomb diamond of JJ1. The variations of $\Gamma$ with the backgate $V_{\mathrm{BG}}$ are quite sizeable (up to 20\%), according to the exponential dependence of the Kondo scale (2), and they constitute a central piece of the analysis in the superconducting state, allowing us to span a large part of the phase diagram of the $0-\pi$ transition. We also stress that changing the local sidegates not only allows us to tune the energy levels in the dots, as is clear in Fig. 1(b), but also modifies the hybridization $\Gamma$. The complete evolution of $\Gamma$ with backgate and sidegate voltages can be tracked by the analysis of the Kondo anomalies using Eq. (2) and leads to a greater range of variations (up to $50 \%$ ).

\section{EXPERIMENTAL STUDY OF THE FIRST-ORDER $0-\pi$ TRANSITION}

Having characterized the normal-state properties of our device, we now focus on the superconducting behavior of the nano-SQUID. Figure 2(d) shows typical voltagecurrent characteristics obtained at three arbitrary gate voltages in the superconducting state. For all set points that we have measured, the nano-SQUID shows an abrupt transition to the finite-voltage branch indicating an underdamped device, with hysteretic voltage-current characteristics $[2,4]$. The current at which this sharp jump occurs defines the switching current $I_{\mathrm{sw}}$, which can be precisely determined via a digital filter [31] by calculating the maximum variance of the measured DC voltage; see Appendix C 1. Switching currents of approximately 3 pA up to a few nA can thus be detected in a fully automated fashion.

\section{A. Comparison between valence-induced and Kondo-induced 0- $\pi$ transition}

Here we focus on a comparison of the $0-\pi$ transition behavior in two different correlation regimes, achieved in two distinct regions of the sidegate checkerboard diagram. Figures 4(a) and 4(d) show both operating regions: (a) corresponds to the situation already studied in the normal state, where fully developed Kondo correlations take place for the odd-charge region of JJ1, while (d) reveals an odd-charge state of JJ1, where Kondo correlations do not arise. (The Kondo temperature is lower than the base temperature of the cryostat.) These distinct physical regimes are similarly witnessed in Figs. 4(b) and 4(e), which show the normal-state-conductance trace along the white line in the conductance maps. In panel (b), the Coulomb blockade is fully overcome by the Kondo effect in the odd-charge region of JJ1, while in panel (e), the Coulomb blockade is robust throughout the entire gate range. The most interesting comparison between the two regimes occurs in the superconducting state [see Figs. 4(c) and 4(f)]. In panel (c), a supercurrent reversal [indicated by the crossing of the two curves associated with two different magnetic fluxes as seen in Eq. (1) and Appendix C 2] 

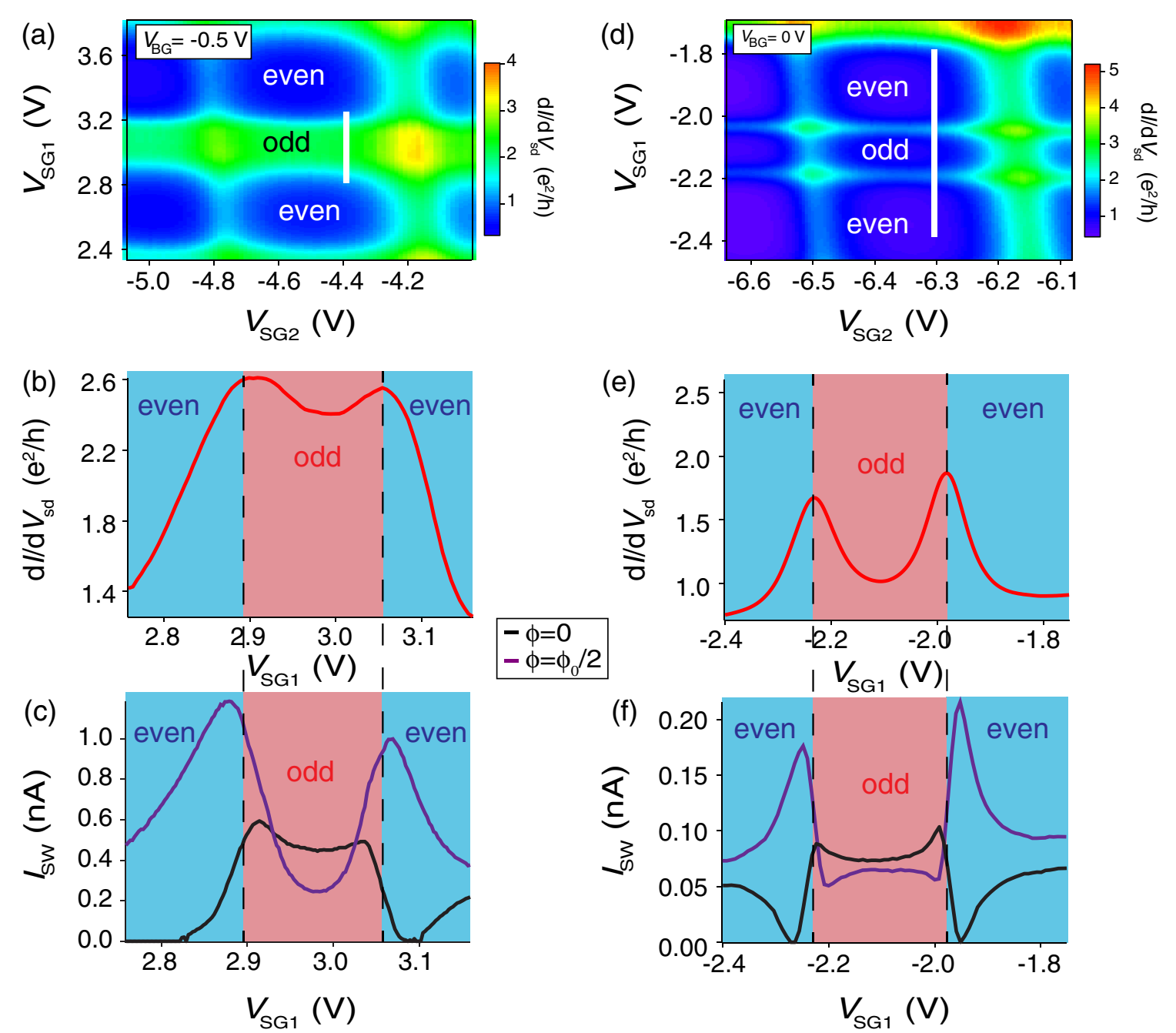

FIG. 4. 0- $\pi$ transition in Kondo- and Coulomb-blockaded odd-charge states. Panels (a), (b), and (c) show, respectively, the stability diagram, the normal-state conductance along the white line in (a), and the supercurrent, in the case of a well-developed Kondo effect. Panels (d), (e), and (f) show, respectively, the stability diagram, the normal-state conductance along the white line in (d), and the supercurrent, in the case when Coulomb blockade is not overcome by the Kondo effect. Supercurrent sign reversal, observed by the crossing of the two curves at $\phi=0$ and $\phi=\phi_{0} / 2$, respectively, penetrates in (c) deep within the odd-charge Coulomb diamond thanks to Kondo screening, in contrast to (f), where the $0-\pi$ crossing occurs precisely when the valence changes from even to odd and from odd to even.

occurs within the odd-charge state of JJ1, showing that the Kondo effect plays a crucial role in triggering the $0-\pi$ transition. In contrast, panel (f) shows that the supercurrent changes sign concomitantly with the increase of the valence of the dot, in agreement with expectations in the strong-Coulomb-blockade regime [29].

\section{B. Tuning the $0-\pi$ transition with controlled changes in the Kondo temperature}

As shown in Sec. II C, it is possible to tune the hybridization $\Gamma$ with the gates, which we exploit to characterize more globally the $0-\pi$ transition phase boundary. Indeed, we have seen previously that Kondo correlations in JJ1 are strengthened when $V_{\mathrm{BG}}$ goes from $-0.3 \mathrm{~V}$ to $-0.7 \mathrm{~V}$ in the operating region of Fig. 2(a). In order to explore precisely the influence of Kondo correlations on the 0- $\pi$ transition, we present in Fig. 5 six plots of $I_{\mathrm{SW}}$ vs $V_{\mathrm{SG} 1}$ [along the white line in Fig. 2(a)] at different backgate voltages and magnetic fields.

The traces exhibit two high switching-current peaks corresponding to the Coulomb-degeneracy points on the sides of the Kondo ridge in an odd-occupancy valley of QD1. Recording such traces at different magnetic fields provides access to the flux modulation of the switching current in the nano-SQUID. Increasing the magnetic flux $\phi$ from 0 to $\phi_{0} / 2$ leads to a steady decrease of $I_{\mathrm{sw}}$ outside the odd-occupancy region of $\mathrm{JJ} 1$, which corresponds to a standard 0-type behavior $[3,4]$ in the Coulomb-blockaded even-occupancy valleys of QD1; see Eq. (1) and Appendix C 2. The flux dependence of the switching 

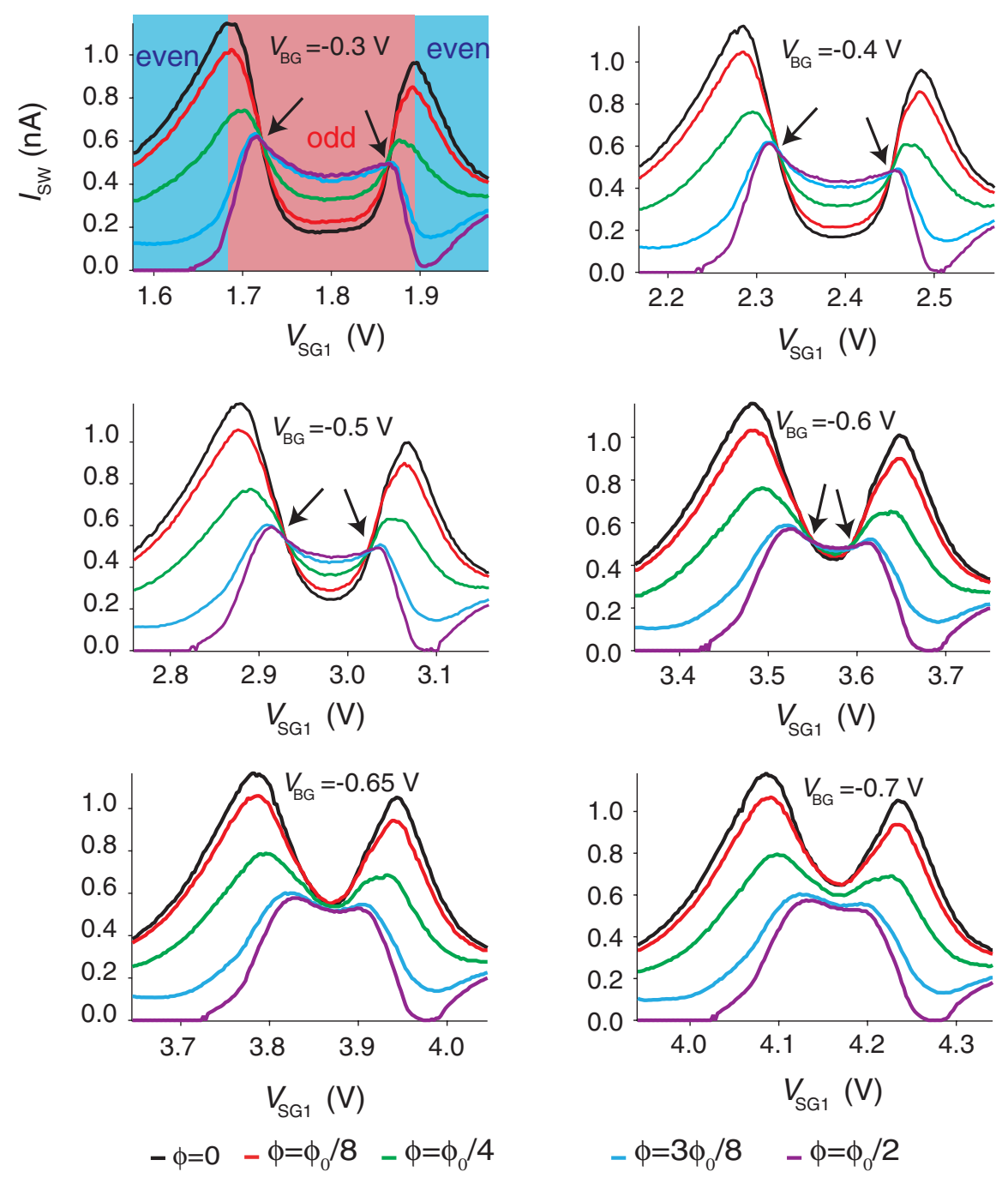

FIG. 5. Switching-current behavior of the nano-SQUID in the Kondo regime. The local gate-voltage $V_{\mathrm{SG} 1}$ dependence of the switching current $I_{\mathrm{sw}}$ is recorded for five magnetic-field strengths (plotted as different colored lines). The panels follow the decrease of the backgate voltage $V_{\mathrm{BG}}$ from $-0.3 \mathrm{~V}$ to $-0.7 \mathrm{~V}$. [The case $V_{\mathrm{BG}}=-0.5 \mathrm{~V}$ is shown in Figs. 4(a)-4(c).] This decrease of the backgate voltage allows us to strengthen the Kondo effect, progressively shrinking the domain associated with $\pi$-junction behavior. Note that, although $V_{\mathrm{SG} 2}$ was corrected for crosstalk in order to stay on the white cut shown in Fig. 2(a), here the actual measured $V_{\mathrm{SG} 1}$ is shown. Arrows denote the transition region between 0 and $\pi$ behavior, associated with the crossing point of the switching current.

current within the odd-charge Kondo domain turns out to be more interesting, as we will analyze next. Clearly, the magnetic-field dependence of $I_{\mathrm{sw}}$ is reversed deep inside the odd-occupancy region of QD1, as the switching current is greater for $\phi=\phi_{0} / 2$ than for $\phi=0$, indicating a $\pi$-type Josephson behavior. One can therefore identify precisely, from the crossing of the switching-current traces, at which sidegate voltage $V_{\mathrm{SG} 1}$ (related to the dot energy) the behavior changes from 0 to $\pi$ type. This allows us to define a $0-\pi$ phase boundary for a given backgate voltage. Now, by similarly examining the $I_{\mathrm{sw}}$ characteristics at different backgate voltages (which allows us to tune the linewidth $\Gamma$ ), we note that decreasing the backgate voltage (i.e., enhancing $\Gamma$ ) reduces the range for $\pi$ behavior, until the $\pi$ phase completely collapses below the critical $V_{\mathrm{BG}}=$ $-0.65 \mathrm{~V}$, and a 0 junction is maintained all along the
Kondo ridge. This physical behavior can be expected from the stronger Kondo screening at larger $\Gamma$ that tends to favor the 0 state. From these measurements, we can unambiguously assign a 0 or $\pi$ behavior to the $\mathrm{JJ} 1$, as a function of both the level position $\epsilon_{0}$ and the width $\Gamma$ of QD1, as determined previously from the analysis of the normal-state transport data. For all recorded transitions (corresponding to the black arrows in Fig. 5), we extract the corresponding microscopic parameters $\Gamma$ and $\epsilon_{0}$, and plot them on an experimental phase diagram shown in Fig. 6(a).

As a quantitative test of our analysis, we display in Fig. 6(a) the theoretical phase diagram obtained from a self-consistent description of Andreev-bound states [28] for $U / \Delta \approx 10$, which corresponds to the experimentally measured $U \simeq 0.8 \mathrm{meV}$ and $\Delta \simeq 80 \mu \mathrm{eV}$. Error bars take 

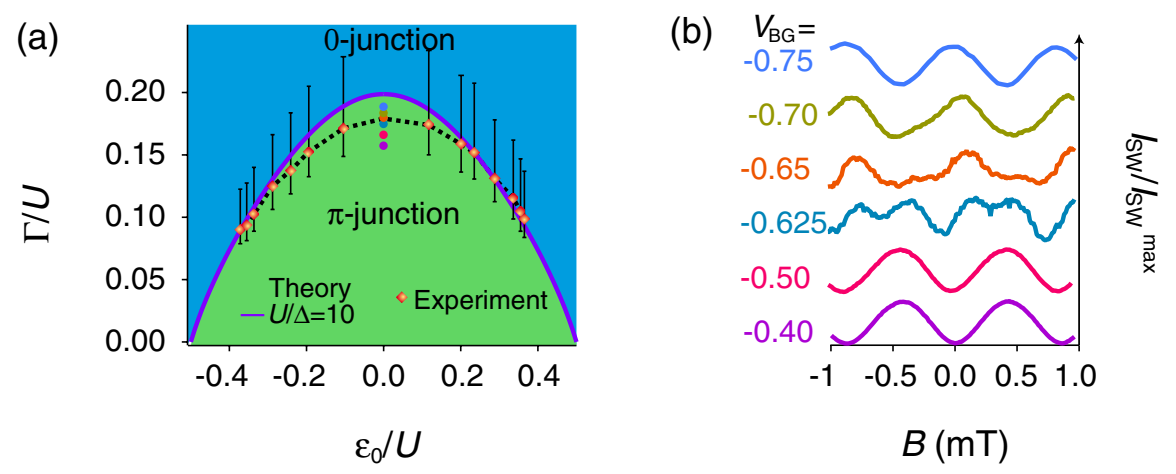

FIG. 6. $0-\pi$ phase transition diagram and nano-SQUID modulations. (a) The experimentally determined phase boundary between 0 - and $\pi$-junction behavior of JJ1 (orange diamonds connected by the dashed line) is given as a function of the dot energy $\epsilon_{0} / U$ and the level width $\Gamma / U$. The Coulomb repulsion $U \approx 0.8 \mathrm{meV}$ is estimated from the finite-bias spectroscopy of the Coulomb-blockade diamond, and $\Delta \approx 80 \mu \mathrm{eV}$ from the proximity effect; see Appendix B. Error bars indicate the uncertainty in the estimate of $\Gamma$ from the analysis of the Kondo resonances. The purple solid line represents the theoretical phase diagram for the experimentally determined ratio $U / \Delta=10$; it slightly overestimates the experimental phase boundary. (b) Magnetic-field modulations of the nano-SQUID switching current taken in the middle of the odd-charge Kondo ridge [shown as the red star in Fig. 2(a)] for different backgate voltages associated with the fine mesh of dots at $\epsilon_{0}=0$ in panel (a). For better comparison, the switching-current modulations are all normalized to the maximum current amplitude, which is strongly suppressed in the transition region. A clear nonharmonic regime occurs near the $0-\pi$ phase boundary, where the bistable behavior (in phase) of the supercurrent may be attributed to the first-order transition between the 0 and $\pi$ states.

into account the uncertainty in the determination of $\Gamma$ from the finite-bias Kondo resonances; see Sec. II C. The bell shape of the phase boundary together with the nearly quantitative agreement with the theory gives strength to the interpretation of the $0-\pi$ transition as a first-order phase transition associated with the crossing of the Andreev-bound states at the Fermi level $[19,26]$. A key point here is that Kondo screening is decisive in allowing the existence of the 0 phase in the center of the odd-charge Coulomb diamond in our experimental conditions $(U \simeq 6 \Gamma)$; see Fig. 7 in Appendix A. While the $0-\pi$ transition is always related to a simple Andreev level crossing, comparison between theory and our data (Fig. 6)

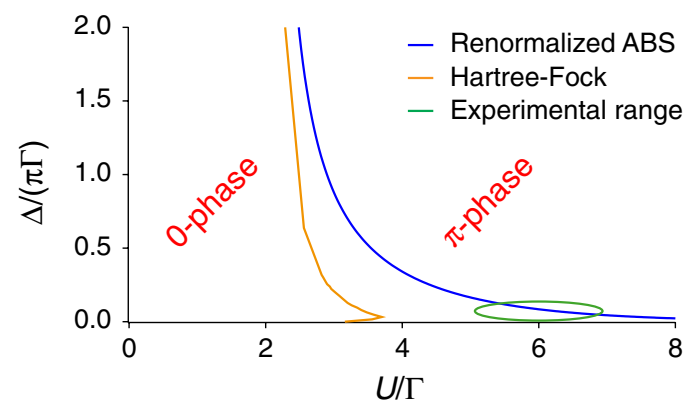

FIG. 7. Theoretical phase diagram for the $0-\pi$ transition at the particle-hole symmetry. We compare the renormalized-ABS theory (which includes Kondo correlations) and the static Hartree-Fock theory. Computations for the large-bandwidth limit of the superconducting Anderson model, Eq. (A1), and the experimental range of the operating region of Figs. 5 and 6, are added for clarity. This comparison shows the key role of Kondo screening in allowing the existence of a $0-\pi$ transition at intermediate correlations $(U>\pi \Gamma)$. clearly demonstrates that it is the competition between the normal-state Kondo temperature $T_{\mathrm{K}}$ and the superconducting gap $\Delta$ that determines the precise location of the $0-\pi$ phase boundary [26,28].

From theoretical expectations [18,27], a second possible smoking gun for the first-order $0-\pi$ phase transition lies in the anharmonic behavior (in phase) of the Josephson junction in close vicinity to the $0-\pi$ phase boundary. This prediction motivates us to consider the field modulation of the switching current $I_{\mathrm{sw}}$ with fine changes of the backgate voltage. This is plotted in Fig. 6(b), where the quantum-dot level is taken in the center of the oddoccupancy Kondo valley [the point of the particle-hole symmetry $\epsilon_{0}=0$, corresponding to the red star in Fig. 2(a) and to the fine mesh of dots in Fig. 6(a)]. For $V_{\mathrm{BG}}>-0.6 \mathrm{~V}$, modulations show that JJ1 is a $\pi$ junction because of the $\phi_{0} / 2$ shift. In contrast, for $V_{\mathrm{BG}}<-0.7 \mathrm{~V}$, modulations turn back to the standard behavior, indicating that $\mathrm{JJ} 1$ is a 0 junction. However, for $-0.6>V_{\mathrm{BG}}>-0.7$, the nano-SQUID switching-current modulations show strong anharmonicities. For this range of backgate voltages, the critical current of JJ1 is very small, leading to a strongly asymmetric SQUID; thus JJ2 always switches at the same phase difference, implying that $I_{\mathrm{sw}}$ directly reflects the current-phase relation of JJ1 [37]. The observed nonharmonic signal can be interpreted as a further indication of the bistable behavior of the junction associated with the first-order $0-\pi$ transition [18-20,23,27].

\section{CONCLUSION}

In conclusion, we have realized a nano-SQUID based on superconducting carbon-nanotube quantum dots. It is fully 
tunable thanks to a set of electrostatic gates, allowing a precise control of the microscopic parameters of the device. This allows us to determine an experimental phase diagram for the $0-\pi$ transition in the Kondo regime, which is in good agreement with theoretical calculations based on the competition between the Kondo temperature and the superconducting gap. The observation of anharmonic behavior in the supercurrent phase relation near the phase boundary is consistent with the first-order nature of the $0-\pi$ transition associated with the crossing of Andreev levels. Fascinating prospects offered by this work are the control and monitoring of the $0-\pi$ transition from supercurrent measurements, as performed here, with simultaneous local spectroscopy of the Andreev spectrum on the quantum dots in the spirit of the recent measurements of Pillet et al. [38]. Such future developments of our experiment, which should be achievable by probing the nano-SQUID with a scanning tunneling microscope, would bring strongly correlated superconducting nanostructures to a new level of control and understanding.

\section{ACKNOWLEDGMENTS}

We thank T. Crozes, E. Eyraud, D. Lepoittevin, C. Hoarau, and R. Haettel for their technical work, C. Thirion and R. Piquerel for their contributions in programming, and V. Bouchiat, H. Bouchiat, C. Balseiro, J.-P. Cleuziou, L. Bogani, S. Datta, D. Feinberg, and T. Novotný for useful discussions. The samples were fabricated in the NANOFAB facility of the Néel Institute. This work is partially financed by ANR-PNANO projects MolNanoSpin No. ANR-08-NANO-002, ERC Advanced Grant MolNanoSpin No. 226558, and STEP MolSpinQIP.

\section{APPENDIX A: THEORETICAL ANALYSIS OF THE 0- $\pi$ PHASE DIAGRAM}

\section{Model for a superconducting quantum dot}

The standard Hamiltonian for describing a single superconducting quantum dot is given by the superconducting Anderson model,

$$
H=\sum_{i=L, R} H_{i}+H_{d}+\sum_{i=L, R} H_{T_{i}},
$$

where

$$
\begin{aligned}
H_{i} & =\sum_{\vec{k}, \sigma} \epsilon_{\vec{k}} c_{\vec{k}, \sigma, i}^{\dagger} c_{\vec{k}, \sigma, i}-\sum_{\vec{k}}\left(\Delta_{i} c_{\vec{k}, \uparrow, i}^{\dagger} c_{-\vec{k} \downarrow, i}^{\dagger}+\text { h.c. }\right), \\
H_{d} & =\sum_{\sigma}\left(\epsilon_{0}+U / 2\right) d_{\sigma}^{\dagger} d_{\sigma}+U n_{\uparrow} n_{\downarrow}, \\
H_{T_{i}} & =\sum_{\vec{k}, \sigma}\left(t d_{\sigma}^{\dagger} c_{\vec{k}, \sigma, i}+\text { h.c. }\right) .
\end{aligned}
$$

In the above equations, $d_{\sigma}$ is the annihilation operator of an electron with spin $\sigma$ on the dot; $c_{\vec{k}, \sigma, i}$ the annihilation operator of an electron with spin $\sigma$ and the wave vector $\vec{k}$ in the lead $i=L, R$; and $n_{\sigma}=d_{\sigma}^{\dagger} d_{\sigma}$. The leads are described by standard s-wave BCS Hamiltonians $H_{i}$ with superconducting gaps $\Delta_{i}=\Delta e^{i \varphi_{i}}$. The phase difference between left and right leads is noted as $\varphi=\varphi_{\mathrm{L}}-\varphi_{\mathrm{R}}$. Furthermore, the leads are assumed to have flat and symmetric conduction bands, i.e., the kinetic energy $\epsilon_{\vec{k}, i}$ measured from the Fermi level ranges in $[-D, D]$, and the density of states is $\rho_{0}=1 /(2 D)$. We assume $\vec{k}$-independent and symmetric tunneling amplitudes $t$ between the dot and both superconducting leads. The dot is described by a single energy level $\epsilon_{0}$ submitted to the Coulomb interaction $U$. (In our convention, $\epsilon_{0}$ vanishes at the center of the Coulomb diamond.)

\section{Renormalized Andreev-bound states and phase diagram of the $0-\pi$ transition}

In the superconducting state, the four atomic states of the quantum dot evolve into renormalized Andreev-bound states (ABS) that possibly live within the gap. A quantitative description of this process was proposed in Ref. [28], beginning with bare values of the ABS splitting in the limit of infinite gap:

$$
\begin{aligned}
& \delta E_{-}^{0}=E_{-}^{0}-E_{\sigma}^{0}=\frac{U}{2}-\sqrt{\epsilon_{0}^{2}+\Gamma_{\varphi}^{2}} \\
& \delta E_{+}^{0}=E_{+}^{0}-E_{\sigma}^{0}=\frac{U}{2}+\sqrt{\epsilon_{0}^{2}+\Gamma_{\varphi}^{2}}
\end{aligned}
$$

with

$$
\Gamma_{\varphi}=\Gamma \frac{2}{\pi} \arctan \left(\frac{D}{\Delta}\right) \cos \left(\frac{\varphi}{2}\right)
$$

In this simplified (and unrealistic) limit, the $0-\pi$ transition corresponds to the crossing of the $|-\rangle$ and $|\sigma\rangle$ states, which occurs for $\delta E_{-}^{0}=0$, leading to a domelike shape in the $\left(\epsilon_{0} / U-\Gamma / U\right)$ plane. However, the phase boundary quantitatively depends on the precise value of the superconducting gap $\Delta$, which must be more realistically included in the calculation. This is done by calculating the corrections at order $1 / \Delta$ to the ABS positions [28], followed by a self-consistency loop that takes into account the leading logarithmic singularities:

$$
\begin{aligned}
\delta E_{-}(\Delta)= & \delta E_{-}^{0}-\frac{\Gamma}{\pi} \int_{0}^{D} d \epsilon\left[\frac{2}{E-\delta E_{-}(\Delta)}-\frac{1}{E+\delta E_{+}^{0}}\right. \\
& -\frac{1}{E+\delta E_{-}^{0}}+\frac{2 \Delta}{E} u v\left|\cos \left(\frac{\varphi}{2}\right)\right|\left(\frac{2}{E-\delta E_{-}(\Delta)}\right. \\
& \left.\left.-\frac{1}{E+\delta E_{+}^{0}}+\frac{1}{E+\delta E_{-}^{0}}\right)\right]+2\left|\Gamma_{\varphi}\right| u v \quad \text { (A5) }
\end{aligned}
$$

and 


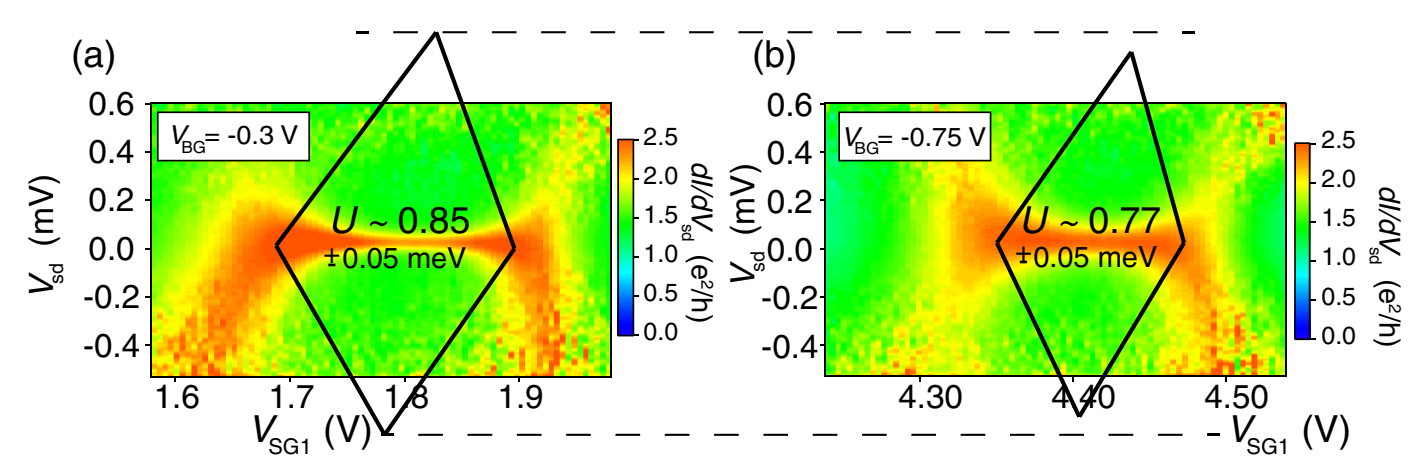

FIG. 8. Conductance map of JJ1 for two values of the backgate voltage. Extending the diamond edges (black lines) to finite bias allows us to extract the Coulomb repulsion of the dot $U=0.80 \pm 0.05 \mathrm{meV}$, where $2 U$ is defined as the energy separation between the two horizontal dashed lines.

$$
\begin{aligned}
\delta E_{+}(\Delta)= & \delta E_{+}^{0}-\frac{\Gamma}{\pi} \int_{0}^{D} d \epsilon\left[\frac{2}{E-\delta E_{+}(\Delta)}-\frac{1}{E+\delta E_{+}^{0}}\right. \\
& -\frac{1}{E+\delta E_{-}^{0}}+\frac{2 \Delta}{E} u v\left|\cos \left(\frac{\varphi}{2}\right)\right|\left(\frac{-2}{E-\delta E_{+}(\Delta)}\right. \\
& \left.\left.-\frac{1}{E+\delta E_{+}^{0}}+\frac{1}{E+\delta E_{-}^{0}}\right)\right]-2\left|\Gamma_{\varphi}\right| u v, \quad(\mathrm{~A} 6)
\end{aligned}
$$

with $E=\sqrt{\epsilon^{2}+\Delta^{2}}$, and where $\delta E_{-}^{0}, \delta E_{+}^{0}$ have been defined in Eqs. (A2) and (A3). The numerical resolution of the self-consistent equation (A5) provides an accurate determination of the phase boundary under the condition $\delta E_{-}(\Delta)=0$, which we successfully compared to the experimental data. Note that we correct here a misprint in Ref. [28], namely, a factor 2 that is missing in front of the second term within the integral in (A5) and (A6).

To stress the key role of the Kondo effect for the 0- $\pi$ transition in our experimental conditions, we compare the phase diagram at the particle-hole symmetry $\left(\epsilon_{0}=0\right)$ obtained from the renormalized-ABS theory [28] and from static Hartree-Fock mean-field theory [18]. (See Fig. 7.) Because the renormalized-ABS approach includes the Kondo scale (at one-loop order), this approach allows the extension of the $0-\pi$ boundary for arbitrary large values of $U / \Gamma$. In contrast, the static mean-field approach is unable to restore a 0 state for Coulomb interaction such that $U \gtrsim \pi \Gamma$, and it fails to reproduce our experimental observation of a supercurrent reversal in the regime $U \simeq 6 \Gamma$. This comparison shows that the phase boundary in our experiment is indeed associated with a competition between the normal-state Kondo temperature and the superconducting gap, in agreement with theoretical expectations $[26,28]$.

\section{APPENDIX B: DETERMINATION OF THE MICROSCOPIC PARAMETERS OF THE NANO-SQUID}

\section{Charging energy}

Because the charging energy $U$ in a carbon-nanotube quantum dot results from the confinement between fixed contacts, one does not expect large variations of $U$ for a small detuning of the backgate. In order to determine the experimental phase diagram for the $0-\pi$ transition, an estimate of $U$ is required. We obtain it by considering the Coulomb-stability diagram of JJ1 for two values of the backgate (see Fig. 8) and extrapolating the diamond edges to large bias.

\section{Proximity gap}

We have performed current-bias measurements to directly access both the superconducting switching current and the differential conductance of the nano-SQUID at $T=35 \mathrm{mK}$. In the presence of superconductivity, the two cotunneling peaks associated with the quasiparticle current in the differential conductance [10] appear at $V= \pm 2 \Delta / e \approx \pm 160 \mu \mathrm{V}$, where $2 \Delta$ is the superconducting gap provided by the proximity effect on the nanotube. (See Fig. 9.) This measurement allows us to extract the superconducting gap in our device, $\Delta \approx 80 \mu \mathrm{eV}$, which is reduced from the bulk value of $\Delta_{\text {bulk }}=175 \mu \mathrm{eV}$ for aluminum, due to the thin palladium contact layer between the carbon nanotube and the aluminum electrodes.

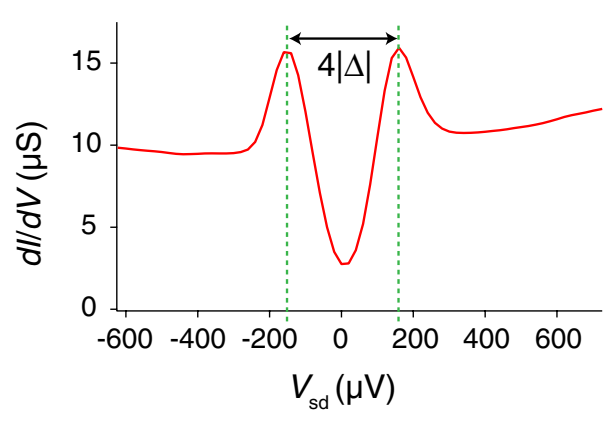

FIG. 9. Signature of the superconducting 50-nm-thick aluminum leads. The differential conductance vs bias voltage shows the presence of a superconducting gap around zero bias, as well as the cotunneling peaks arising from the quasiparticle tunneling. The gap value $\Delta \approx 80 \mu \mathrm{eV}$ is thus obtained from the $4 \Delta$-wide gap denoted by the double arrow. The measurement is performed in a blocked region of the nano-SQUID, which explains why no supercurrent is visible at zero bias. 

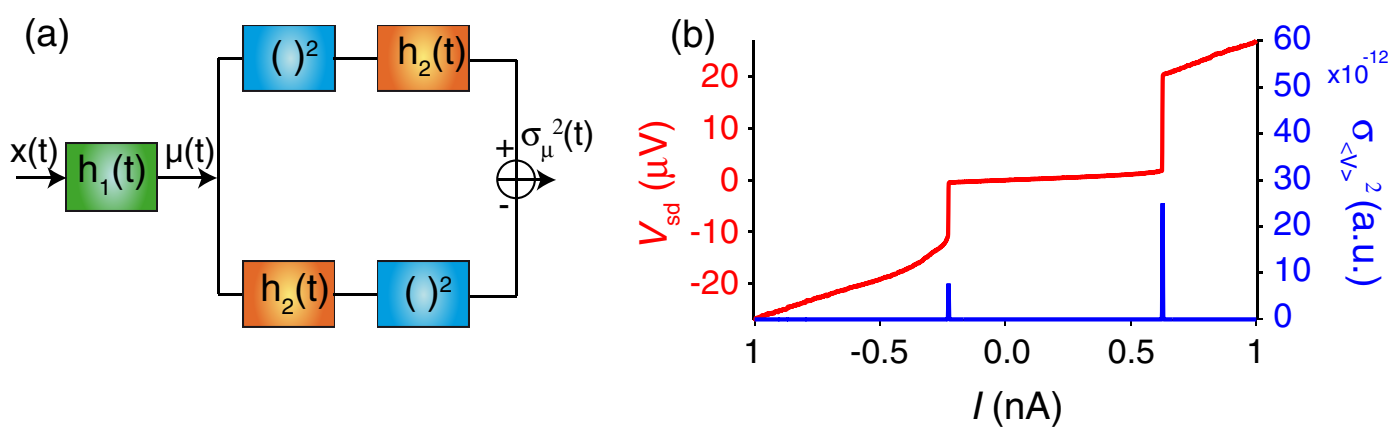

FIG. 10. Digital filter for switching-current detection. (a) Schematic view of the implemented filter based on the work of Liu et al. [31]. The first-order moment $\mu(t)$ of the signal $x(t)$ in a sliding window is obtained through the filter $h_{1}(t)=\operatorname{Rect}\left(t / L_{1}\right) / L_{1}$, where $\operatorname{Rect}(t)$ is the normalized rectangular function and $L_{1}$ is the filter length. The variance $\sigma_{\mu}^{2}(t)$ of $\mu(t)$ is simply calculated as $\left\langle\mu(t)^{2}\right\rangle-\langle\mu(t)\rangle^{2}$ in a sliding window with $h_{2}(t)=\operatorname{Rect}\left(t / L_{2}\right) / L_{2}$. (b) Voltage-current characteristics and the estimated variance of the first-order moment obtained by the implemented digital filter.

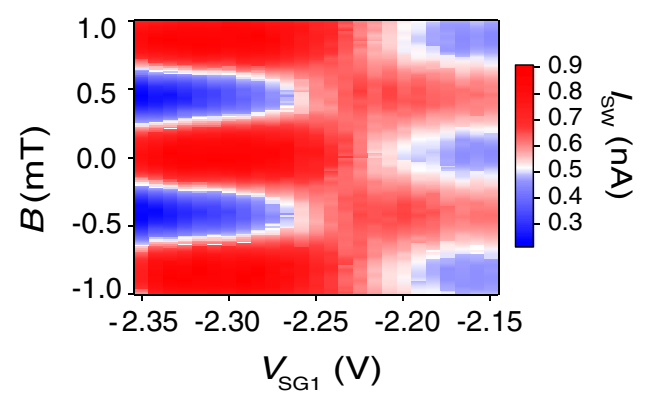

FIG. 11. Complete modulation of the switching current by the applied magnetic field around the $0-\pi$ transition. $I_{\mathrm{SW}}$ vs magnetic field and sidegate voltage $V_{\mathrm{SG} 1}$ around the $0-\pi$ transition in JJ1, corresponding to the trace in Figs. 4(d)-4(f). The 0 state is stabilized for $V_{\mathrm{SG} 1}<-2.25 \mathrm{~V}$, while the $\pi$ state occurs for $V_{\mathrm{SG} 1}>-2.20 \mathrm{~V}$. Nonharmonic behavior of $I_{\mathrm{SW}}$ as a function of magnetic flux is clearly observed in the intermediate transition range.

\section{APPENDIX C: SWITCHING-CURRENT DETECTION AND SWITCHING-CURRENT MAGNETIC-FIELD MODULATION}

\section{Switching-current detection}

To have an accurate method of extracting the switching current from voltage/current characteristics, even for small transition voltage jumps to the dissipative state, we have implemented a digital filter based on the work of Liu et al. [31]. The main purpose of this filter is the detection of transitions from a noisy signal, which we apply to the superconducting/normal transition. The operation consists of estimating the variance of the firstorder moment of the signal in a sliding window. A schematic view of the filter is presented in Fig. 10(a). To begin, the first-order moment $\mu(t)$ is estimated via a classic averaging filter in a sliding window characterized by the impulse response $h_{1}(t)=\operatorname{Rect}\left(t / L_{1}\right) / L_{1}$, where $\operatorname{Rect}(t)$ is the normalized rectangular function and $L_{1}$ is the filter length. Finally, the estimated variance is obtained by $\left\langle\mu(t)^{2}\right\rangle-\langle\mu(t)\rangle^{2}$ with another averaging filter $h_{2}(t)$ of length $L_{2}$. For the switching-current detection with a sample rate of 1000 , we have taken $L_{1}=L_{2}=$ 4. Such a filter provides a sharp signal from the steplike features of our voltage/current characteristics, as presented in Fig. 10(b).

\section{Switching-current magnetic-field modulation around the $0-\pi$ transition}

The main experimental signature of a transition between a 0 junction and a $\pi$ junction is the $\pi$ shift that occurs in the switching-current magnetic-field modulation; see Eq. (1). To reveal this phase shift, one can display the switching current vs backgate or sidegate voltage at two different magnetic fields corresponding to fluxes in the nano-SQUID loop of, respectively, $\phi=0$ and $\phi=\phi_{0} / 2$, as presented in the main paper. A more systematic illustration is given here by the complete switching-current magnetic-field modulation recorded around the $0-\pi$ transition, as shown in Fig. 11.

[1] S. De Franceschi, L. Kouwenhoven, C. Schönenberger, and W. Wernsdorfer, Hybrid Superconductor-Quantum Dot Devices, Nature Nanotech. 5, 703 (2010).

[2] P. Jarillo-Herrero, J. A. Van Dam, and L. P. Kouwenhoven, Quantum Supercurrent Transistors in Carbon Nanotubes, Nature (London) 439, 953 (2006).

[3] J. A. Van Dam, Y. V. Nazarov, E. P. A. M. Bakkers, S. De Franceschi, and L. P. Kouwenhoven, Supercurrent Reversal in Quantum Dots, Nature (London) 442, 667 (2006).

[4] J.-P. Cleuziou, W. Wernsdorfer, V. Bouchiat, T. Ondarçuhu, and M. Monthioux, Carbon Nanotube Superconducting Quantum Interference Device, Nature Nanotech. 1, 53 (2006).

[5] C. B. Winkelmann, N. Roch, W. Wernsdorfer, V. Bouchiat, and F. Balestro, Superconductivity in a Single $\mathrm{C}_{60}$ Transistor, Nature Phys. 5, 876 (2009). 
[6] L. Hofstetter, S. Csonka, J. Nygård, and C. Schönenberger, Cooper Pair Splitter Realized in a Two-Quantum-Dot Y-Junction, Nature (London) 461, 960 (2009).

[7] L. G. Herrmann, F. Portier, P. Roche, A. Levy Yeyati, T. Kontos, and C. Strunk, Carbon Nanotubes as Cooper-Pair Beam Splitters, Phys. Rev. Lett. 104, 026801 (2010).

[8] H. Ingerslev Jorgensen, T. Novotný, K. Grove-Rasmussen, K. Flensberg, and P.E Lindelof, Critical Current O-pi Transition in Designed Josephson Quantum Dot Junctions, Nano Lett. 7, 2441 (2007).

[9] A. Y. Kasumov, R. Deblock, M. Kociak, B. Reulet, H. Bouchiat, I. I. Khodos, Yu. B. Gorbatov, V.T. Volkov, C. Journet, and M. Burghard, Supercurrents through SingleWalled Carbon Nanotubes, Science 284, 1508 (1999).

[10] M. R. Buitelaar, T. Nussbaumer, and C. Schonenberger, Quantum Dot in the Kondo Regime Coupled to Superconductors, Phys. Rev. Lett. 89, 256801 (2002).

[11] J.-P. Cleuziou, W. Wernsdorfer, V. Bouchiat, T. Ondarçuhu, and M. Monthioux, Tuning the Kondo Effect with Back and Side Gates-Application to Carbon Nanotube Superconducting Quantum Interference Devices and Pi-Junctions, arXiv:cond-mat/0610622v1.

[12] J.-P. Cleuziou, W. Wernsdorfer, S. Andergassen, S. Florens, V. Bouchiat, T. Ondarçuhu, and M. Monthioux, Gate-Tuned High Frequency Response of Carbon Nanotube Josephson Junctions, Phys. Rev. Lett. 99, 117001 (2007).

[13] E. Pallecchi, M. Gaass, D. A. Ryndyk, and Ch. Strunk, Carbon Nanotube Josephson Junctions with $\mathrm{Nb}$ Contacts, Appl. Phys. Lett. 93, 072501 (2008).

[14] K. Grove-Rasmussen, H. I. Jørgensen, B. M. Andersen, J. Paaske, T. S. Jespersen, J. Nygård, K. Flensberg, and P. E. Lindelof, Superconductivity-Enhanced Bias Spectroscopy in Carbon Nanotube Quantum Dots, Phys. Rev. B 79, 134518 (2009).

[15] A. Eichler, R. Deblock, M. Weiss, C. Karrasch, V. Meden, C. Schonenberger, and H. Bouchiat, Tuning the Josephson Current in Carbon Nanotubes with the Kondo Effect, Phys. Rev. B 79, 161407 (2009).

[16] Y. Kanai, R. S. Deacon, A. Oiwa, K. Yoshida, K. Shibata, K. Hirakawa, and S. Tarucha, Electrical Control of Kondo Effect and Superconducting Transport in a Side-Gated InAs Quantum Dot Josephson Junction, Phys. Rev. B 82, 054512 (2010).

[17] C. W. J. Beenakker and H. van Houten, Single-Electron Tunneling and Mesoscopic Devices (Springer, Berlin, 1992).

[18] A. V. Rozhkov and D.P Arovas, Josephson Coupling through a Magnetic Impurity, Phys. Rev. Lett. 82, 2788 (1999).

[19] A. A. Clerk and V. Ambegaokar, Loss of $\pi$-Junction Behavior in an Interacting Impurity Josephson Junction, Phys. Rev. B 61, 9109 (2000).

[20] T. Yoshioka and Y. Ohashi, Numerical Renormalization Group Studies on Single Impurity Anderson Model in Superconductivity: A Unified Treatment of Magnetic, Nonmagnetic Impurities, and Resonance Scattering, J. Phys. Soc. Jpn. 69, 1812 (2000).
[21] E. Vecino, A. Martin-Rodero, and A. Levy Yeyati, Josephson Current through a Correlated Quantum Level: Andreev States and $\pi$ Junction Behavior Phys. Rev. B 68, 035105 (2003).

[22] F. Siano and R. Egger, Josephson Current through a Nanoscale Magnetic Quantum Dot, Phys. Rev. Lett. 93, 047002 (2004).

[23] M. S. Choi, M. Lee, K. Kang, and W. Belzig, Kondo Effect and Josephson Current through a Quantum Dot between Two Superconductors, Phys. Rev. B 70, 020502 (2004).

[24] G. Sellier, T. Kopp, J. Kroha, and Y. S. Barash, $\pi$-Junction Behavior and Andreev Bound States in Kondo Quantum Dots with Superconducting Leads, Phys. Rev. B 72, 174502 (2005).

[25] T. Novotný, A. Rossini, and K. Flensberg, Josephson Current through a Molecular Transistor in a Dissipative Environment, Phys. Rev. B 72, 224502 (2005).

[26] J. Bauer, A. Oguri, and A. C. Hewson, Spectral Properties of Locally Correlated Electrons in a Bardeen-CooperSchrieffer Superconductor, J. Phys. Condens. Matter 19, 486211 (2007).

[27] C. Karrasch, A. Oguri, and V. Meden, Josephson Current through a Single Anderson Impurity Coupled to BCS Leads, Phys. Rev. B 77, 024517 (2008).

[28] T. Meng, S. Florens, and P. Simon, Self-Consistent Description of Andreev Bound States in Josephson Quantum Dot Devices, Phys. Rev. B 79, 224521 (2009).

[29] L. I. Glazman and K. A. Matveev, Resonant Josephson Current through Kondo Impurities in a Tunnel Barrier, JETP Lett. 49, 659 (1989) [http://www.jetpletters.ac.ru/ps/ 1121/article_16988.shtml].

[30] L. Kouwenhoven and L. Glazman, Revival of the Kondo Effect, Phys. World 14, 33 (2001).

[31] W. Y. Liu, I. E. Magnin, and G. Gimenez, A New Operator for the Detection of Transitions in Noisy Signals, Traitement du Signal 12, 225 (1995) [http://hdl.handle .net/2042/1907, in French].

[32] H. Grabert and M.H. Devoret, Single Charge Tunneling (Plenum Press, New York, 1992).

[33] D. Goldhaber-Gordon, J. Göres, M. Kastner, Hadas Shtrikman, D. Mahalu, and U. Meirav, From the Kondo Regime to the Mixed-Valence Regime in a Single-Electron Transistor, Phys. Rev. Lett. 81, 5225 (1998).

[34] F.D. M. Haldane, Scaling Theory of the Asymmetric Anderson Model, Phys. Rev. Lett. 40, 416 (1978).

[35] R. Bulla, T. A.C. Costi, and T. Pruschke, Numerical Renormalization Group Method for Quantum Impurity Systems, Rev. Mod. Phys. 80, 395 (2008).

[36] T. Fujii and K. Ueda, Perturbative Approach to the Nonequilibrium Kondo Effect in a Quantum Dot, Phys. Rev. B 68, 1 (2003).

[37] M. Della Rocca, M. Chauvin, B. Huard, H. Pothier, D. Esteve, and C. Urbina, Measurement of the Current-Phase Relation of Superconducting Atomic Contacts, Phys. Rev. Lett. 99, 127005 (2007).

[38] J.-D. Pillet, C.H. L. Quay, P. Morfin, C. Bena, A. Levy Yeyati, and P. Joyez, Andreev Bound States in Supercurrent-Carrying Carbon Nanotubes Revealed, Nature Phys. 6, 965 (2010). 